\title{
How the Strength of a Strong Object Mask Varies in Space and Time When It Is Used as an Uninformative Singleton in Visual Search for Target Location ${ }^{*}$
}

\author{
Talis Bachmann ${ }^{1}$, Endel Põder ${ }^{2}$, Carolina Murd ${ }^{1}$ \\ ${ }^{1}$ Institute of Public Law, University of Tartu, Tallinn, Estonia; \\ ${ }^{2}$ Department of Psychology, University of Tartu, Tartu, Estonia. \\ Email: talis.bachmann@ut.ee \\ Received August 26 $6^{\text {th }}, 2011$; revised September $28^{\text {th }}$, 2011; accepted October $29^{\text {th }}, 2011$.
}

\begin{abstract}
Strong visual masking originates from sensory perceptual interactions between target and mask and also from attentional competition between target and mask even though mask does not correspond to attentional control settings. The relative contributions of these different masking mechanisms are difficult to estimate. One strategy to begin approach this problem is to use the same stimulus as a mask and as a non-informative singleton in a selective attention task. The purpose of the present study was to find the spatial and temporal intervals where a strong object mask interferes with target-object search when used as a non-informative singleton. In visual search for target location, we found that a visual object that has a strong forward and backward masking power on target-object correct perception when spatially superimposed on target can impair target perception from a spatially separated location only when presented up to $100 \mathrm{~ms}$ after the target and only from a spatially close location. These results are explained by a processing account where the initial analysis of stimuli features allows to determine the best candidate location for the target, but as soon as this location is established, a nearby later appearing object may intrude it, replacing the target in explicit perception. The higher-level mechanisms based interpretation is strengthened by the finding that any local masking effects of the same adjacent singleton were absent in the task of single-target identification.
\end{abstract}

Keywords: Masking, Visual Search, Target Localization, Attentional Capture, Consciousness

\section{Introduction}

\section{Impairment of Perception by Masking and Attentional Miscueing}

Surprisingly perhaps, but there are many ways normal people with normal vision cannot see (Breitmeyer, 2010). Part of the reasons why objects cannot be consciously seen owe to the limitations and interactions of early sensory-perceptual mechanisms; another part of the cases of not seeing takes place because objects may remain unattended or inaccessible for attention. In one of these phenomena called visual masking an otherwise well perceptible brief stimulus object, a target, becomes barely visible or completely invisible if accompanied in space and time by another object-a mask. Masking is a complex phenomenon occurring in different varieties and emerging as an outcome of the effects of early sensory-perceptual mechanisms and/or attentional mechanisms (Bachmann, 1994; Enns, 2004). Thus, strong visual masking originates not only from sensory-perceptual interactions between target and mask, but also from attentional competition between target and mask even though subjects try to ignore the mask as much as they can (Di Lollo, Enns, \& Rensink, 2000; Michaels \& Turvey, 1979; Smith, \& Wolfgang, 2004). The relative contributions of these different masking mechanisms are difficult to estimate. A masking stimulus may at the same time inhibit target's perceptual processing, deprive the target from access to consciousness or draw attention away from target processing. Unfortunately, the power of a stimulus object to act as a mask and act as a cue for capturing attention has been studied in completely different sets of research.

One strategy to begin approach this problem would be to start with setting up experiments where the same stimulus is used as a mask and as a distractor in a selective attention task. In one of its roles, a stimulus different from any target would be used as a visual object-mask covering target location, but presented either before the target (in forward masking) or after the target (in backward masking). In the other of its roles, the same stimulus is presented either not far from the target location (as a valid attentional cue) or far from the target location (as an invalid attentional cue). The selective attention task is that of visual search-a typical task where subjects search for a target stimulus pre-specified before each trial. The main interest would be to compare the relative effectiveness of the same stimulus as a mask and as a bottom-up attentional cue. The relative expression of the effect of the cue/mask object as a mask or as a cue for involuntary attentional capture on target perception can be studied.

It is widely believed that reliable and explicit visual recognition depends critically on whether selective attention can participate in the processing of object information. This standpoint has been repeatedly advocated in the many studies of visual search and attentional cueing (e.g., Cheal, \& Lyon, 1991; Davoli, Suszko, \& Abrams, 2007; Di Lollo, Enns, \& Rensink, 2000; Gibson et al., 2008; Kahneman, \& Treisman, 1984; Kawahara, \& Miyatani, 2001; Müller \& Krummenacher, 2006; Vierck, \& Miller, 2008; Yantis, \& Jonides, 1990). However, it is not unanimously clear whether the perception-improving or impairing selective attention can be attracted also automatically by uninformative singleton objects appearing somewhere in the visual field (Müller \& Krummenacher, 2006; Yeh, \& Liao, 2010). An uninformative singleton that appears spatially close to the target object supposedly draws attention to that generic 
location and improves target processing; the same singleton, if presented in a spatially remote location supposedly acts as an invalid cue and therefore impairs target processing by sending attention to a wrong place. On the other hand, if the perceptualattentional system can concentrate processing on the target and ignore the uninformative singleton, singleton presentation may remain without effect, neither a facilitatory nor a perturbing one. This can hold especially when instead of selective attentional focusing by spatial cueing, selective attention is engaged in tasks of visual target search where attentional processes are preset by target identity and not by spatial location.

It is generally accepted that the likelihood that an uninformative object will effectively shift attention away from a target depends most of all on two factors: 1 ) its relative salience and 2) attentional control settings or task relevance (e.g., Ansorge, \& Horstmann, 2007; Folk, Remington, \& Johnston, 1992; Lichtenstein-Vidne, Henik, \& Safadi, 2007; Müller, \& Krummenacher, 2006; Yantis, \& Jonides, 1990). Even though the controversy over whether spatial attention can be automatically captured when the uninformative singleton is free from being associated with control settings, has not been conclusively solved (for the answer "yes" see, e.g., Davoli, Suszko, \& Abrams, 2007; Forster, \& Lavie, 2008; Gibson, Folk, Theeuwes, \& Kingstone, 2008; Neo, \& Chua, 2006; Turatto, \& Galfano, 2000; Yeh, \& Liao, 2010; for the answer "no" see, e.g., Becker, 2007; Gibson et al., 2008; Yantis, \& Jonides, 1990; Jingling, \& Yeh, 2007), there seems to be at least one agreement. It is believed that if attention is not spatially focused before presentation of a set of alternative objects (including the target), sufficiently salient uninformative singletons typically capture attention in a bottom-up, involuntary manner and may do so in advance of target exposure (Müller, \& Krummenacher, 2006; Neo, \& Chua, 2006). This leads to costs in target discrimination performance. Therefore, in the present research we use visual search where target's spatial position is not known to subjects at the outset of their search trials and they themselves have to figure out that position. As a corollary, we expect involuntary attentional capture.

\section{The Present Approach and Its Aims}

If we compare the many studies carried out on visual search and distracting effects on it, we can easily notice the wide variety of the experimental variables and of the values of the variables used. The problem of distractability of processing in visual covert search cannot be easily solved when the dispute is based on varied sets of data drawn from experiments using different parameters of stimulation-different number of alternatives, types of features, durations of stimuli, stimuli onset asynchronies (positive and/or negative, narrowly selected, time values), sizes of stimuli, spatial distances between stimuli, luminances and contrasts, using masks or not, target-distractor similarity values, number of varying dimensions of features, etc. Often the yes-no type of target detection is used readily allowing non-sensory bias effects; often the dependent measure is reaction time whereby pre-conscious automatic facilitation effects and conscious-level, explicit perception effects cannot be separated (response priming effects can be heavily preconscious-Mulckhuyse, \& Theeuwes, 2010; Van den Bussche et al., 2010). In the present work, we use correct localization of targets among the many alernative positions as the measure of veridical target perception and use one and the same stimulus as the mask and as the uninformative bottom-up cue for attentional capture. In the present study we examine the possible effects of object masks in the role of uninformative singleton objects on visual covert search by systematically varying the spatial and temporal distance between the target and the singleton object over a wide range of spatial and temporal values. We aim to map the target-to-singleton spatial- and temporal-distance values that lead either to improvement or impairment of target processing compared to spatial and temporal intervals that do not help get clear effects. First of all, we aim to assess comparatively the strength of an invariant singleton object used as a mask and used as an uninformative cue. Depending on the results, we will discuss the possible support or inconsistency of the data with regard to known processing mechanisms and theories of attentive perception in target search.

The following simple hypotheses were put forward: 1) When a singleton object as a forward mask is presented optimally ( 0 - $150 \mathrm{~ms})$ in advance of the display that contains a pre-specified search-target object and when it spatially overlaps the target location, strong masking is expected. 2) When a singleton object as a backward mask is presented optimally (0 $150 \mathrm{~ms}$ ) subsequent to the display that contains a pre-specified search-target object and when it spatially overlaps the target location, strong masking is expected. 3) When a singleton object is presented optimally (50 - $150 \mathrm{~ms}$ ) in advance of the display that contains a pre-specified search-target among distractors and when it is spatially close to the target location (a condition of valid spatial-attentional pre-cueing), target correct localization rate will be higher compared to the overall mean localization performance. 4) When a singleton object is presented optimally (50 - $150 \mathrm{~ms}$ ) in advance of the display that contains a pre-specified search-target among distractors and when it is spatially far from the target location (acting as an invalid spatial pre-cue), target correct localization rate will be lower compared to the overall mean localization performance. 5) When a singleton object is presented optimally (50 - $150 \mathrm{~ms}$ ) after the display that contains a pre-specified search-target among distractors and when it is spatially close to the target location, target correct localization rate will be higher compared to the overall mean localization performance-the singleton acting as a valid after-cue aiding selection from sensory visual memory. 6) When a singleton object is presented optimally (50 - $150 \mathrm{~ms}$ ) after the display that contains a pre-specified searchtarget among distractors and when it is spatially far from the target location (acting as an invalid spatial after-cue), target correct localization rate will be lower compared to the overall mean localization performance-visual sensory memory will be selectively mis-cued. In the experiment the singleton object as a mask/cue will be consistently mapped onto different color and pattern features compared to the targets and fillers/distractors (that will be mutually a varied mapping set). If some of the hypotheses will get support, interpretation in favor or against particular attentional processing accounts will depend on the exact combination of the hypotheses that will have received empirical support.

Independent of any particular theory of processing in search, the visual system should solve the following sub-tasks: 1) activate target identity nodes in the visual representation system when target identity is specified before presentation of alternatives, 2) when alternatives consisting in target and distractors have been presented, test alternative object-features from different locations for the match between target features and the perceptual features of an actual object presented from certain spatial locations, 3) when a fitting match is found, register the corresponding spatial location, 4) respond by indicating this location explicitly. 
The singleton we used was chosen so that it remained fully uninformative - it had to have no predictive value with regard to target identity, location or presentation time neither due to sharing its unique features nor because of being presented predictably either before, simultaneously with, or after the target and distractors.

\section{Experiment 1}

The aim of Experiment 1 was to find the spatial and temporal intervals between singleton-object and target object where singleton helps facilitate target processing and where it leads to impairment of target processing in order to test the above described hypotheses. In the within subjects design singletonto-target spatial separation and temporal separation between singleton and target are the main independent variables and rate of correct target localization is the principal dependent variable.

\section{Method}

\section{Participants}

A group of 6 subjects (mean age 23; 3 females, 3 males) participated in the main experiment. They had normal or corrected-to-normal vision; they participated as paid volunteers (180 Estonian Kroon per experiment per subject). In piloting the experiment with fewer trials per subject and using 8 subjects we found qualitatively highly similar data to the data by the main group, therefore the results obtained with 6 observers can be considered as representative.

\section{Stimuli and Apparatus}

Both target and distracting items were selected from the same set of 8 stimuli; thus a varied mapping format was used. The stimuli were Gabor patches with varying colour (purple or green), spatial frequency (2 or 4 cycles/deg) and orientation (vertical or horizontal). Each one of these features was used an equal number of times. As targets were defined by conjunction of several features, pre-attentive filtering could not be used in effective search. Stimuli subtended .62 degrees of visual angle. Eight Gabors (the target and 7 fillers/distractors) were presented in locations forming an imaginary circle (radius 4 deg, with a small jitter) around the fixation dot. The uninformative featuresingleton used as a mask and/or uninformative cue was a yellow ring equal in size to the other 8 stimuli. It was presented on the same imaginary ring, located either as superimposed with possible target locations or placed in a midpoint between two other stimuli. The stimuli were presented on a computer monitor (Eizo Flex-scan T550), refresh rate $85 \mathrm{~Hz}$, on a grey background $\left(57 \mathrm{~cd} / \mathrm{m}^{2}\right)$ with a duration of $24 \mathrm{~ms}$. An example of a stimulus display is given in Figure 1.

\section{Procedure}

Each trial started with presentation of a fixation cross $(.2$ deg). Participants initiated presentation of the stimuli by pressing the Enter key on the keyboard. An example of one of the 8 Gabor-type stimuli then appeared for $500 \mathrm{~ms}$ in the center of the display indicating a target stimulus for that trial. After 1200 $\mathrm{ms}$, one of the three temporal types of trials ocurred: 1) yellow uninformative feature-singleton appearing first, followed by 8 stimuli (1 target, 7 fillers) with SOA varying between $120 \mathrm{~ms}$, $96 \mathrm{~ms}, 72 \mathrm{~ms}, 48 \mathrm{~ms}$, and $24 \mathrm{~ms}$; 2) 8 stimuli were presented first, followed by yellow feature-singleton with SOA varying between $120 \mathrm{~ms}, 96 \mathrm{~ms}, 72 \mathrm{~ms}, 48 \mathrm{~ms}$, and $24 \mathrm{~ms}$; 3) target and distractor fillers presented simultaneously with the yellow feature-singleton, this condition limited to the majority of trials

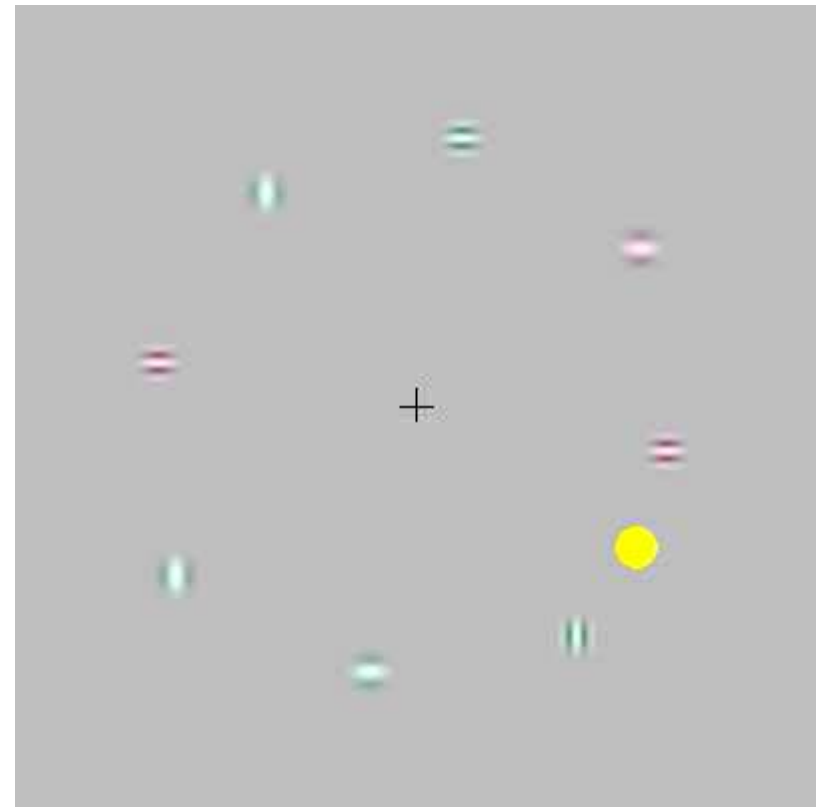

Figure 1.

An example of search display where one of the Gabor-like stimuli is a target prespecified by showing it before this type of display, the other Gabors are fillers/distractors and the yellow disc is a non-informative singleton object.

where singleton occupied a location different from target. Feature-singletons appeared at randomly chosen locations relative to the target on an imaginary ring. Thus spatial distance between the singleton and target varied between $0 \mathrm{deg}, 1.55 \mathrm{deg}$, $3.04 \mathrm{deg}, 4.42 \mathrm{deg}, 5.66 \mathrm{deg}, 6.65 \mathrm{deg}, 7.38 \mathrm{deg}, 7.84 \mathrm{deg}$, and $8.00 \mathrm{deg}$, including the one with spatial separation 0 , i.e., the singleton and the target were presented from the identical spatial position (except when SOA $=0 \mathrm{~ms}$ where the singleton was always in a different location from that of the target). After stimuli presentation, participants indicated by a mouse-click the spatial position where they perceived the target was presented. Then the next trial followed. Each subject ran 1000 trials, with 6,000 trials across six participants.

\section{Results and Discussion}

Repeated measures ANOVA shows that SOA had no significant main effect $(F(9,45)=2.01, p<.06)$. A tendency for lower level of target correct localization performance with increasing SOA can be noticed. Spatial distance (separation) between singleton and target was highly significant $(F(8,40)=$ 23.55, $p<.0001$ ). As can be seen also from Figure 2, this owes first of all to the fact that when mask/cue singleton spatially overlaps with target (separation distance 0 deg), target correct localization level dramatically drops. (Vertical bars denote .95 confidence.) There was no interaction between SOA and spatial separation $(F(73,360)=1.284, p<.074)$. In the condition with spatially superimposed singleton and target (distance 0 ) when singletons acted as forward masks (i.e., negative SOA values were used), the target correct localization level dropped significantly.

Taken together, the basic results are: 1) with most singletonto-target spatial and temporal distances neither facilitative nor interfering effects on target correct localization were found, which does not support hypotheses $3-6$; 2) a strong forwardmasking effect emerged with spatially superimposed singletons 


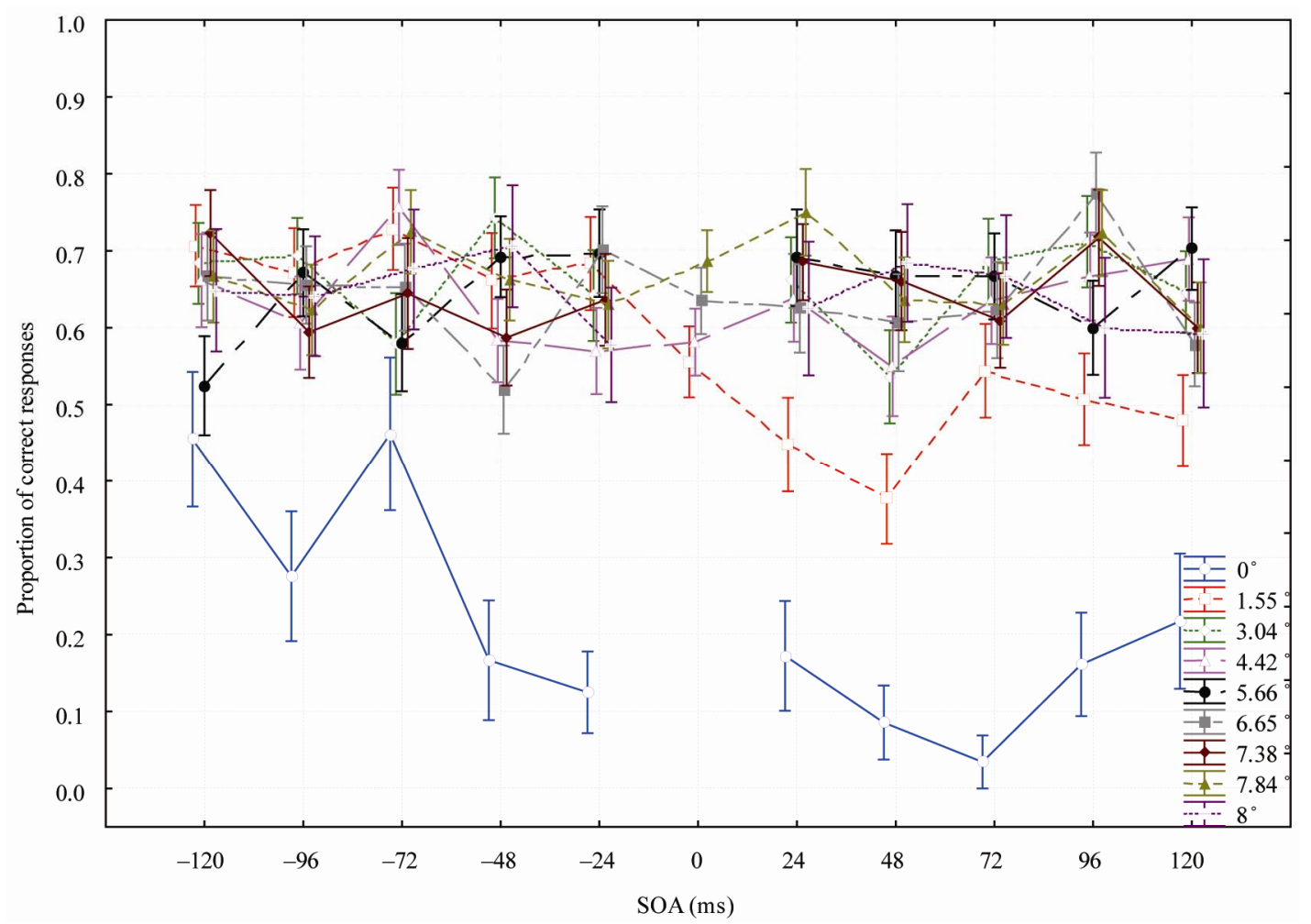

Figure 2.

Proportion of correct target localization responses as a function of SOA between uninformative singleton and target and spatial distance between singleton and target. Singletons spatially superimposed with targets have a strong interfering effect both when presented before and after the targets, with the strongest effect taking place with singletons that followed targets in time. (Data from Experiment 1.)

and targets, supporting hypothesis 1 ; 3) when singletons that followed the targets were presented from the same location, backward-masking effect was strongly evident, supporting hypothesis 2; 4) with the closest spatial separation between the non-overlapping singleton and a target there is some significant singleton effect specifically with later-appearing singletons, which goes against hypothesis 5 and extends backward masking effect to the conditions with clearly spatially separated targets and singleton-as-mask. Spatial pre-cueing from a close target location does not help to enhance correct target processing rate, nor does an invalid type of precueing from locations far from the target produce pre-cueing costs. Interference and masking effects definitely overweighed any putative spatial-attentional valid cueing effects supposed to help target perception in case of spatially close cueing and impair target peception in case of spatially distant, invalid pre-cueing. From these results we can conclude that there is no competition for unspecific general attentional resources between singleton and target, no attentioncapturing effect of the uninformative singletons as pre-cues or after-cues, but a strong forward and backward masking effect when the same singletons are used as forward and backward masks. A novel result shows that a stimulus that is indifferent for search of targets in its role as a spatial-attentional cue acts as a strong visual mask for searched target perception. An uninformative singleton stimulus incapable of engaging effective involuntary bottom-up attention (i.e., a stimulus that can be ignored in searching for the pre-designated feature-conjunction targets) cannot be ignored when used as a spatially overlapping object mask and vice versa. The only condition where the spatially non-overlapping singleton has its effect on correct perception of target location is when it comes after the target and from the spatially closest non-overlapping position. From the attentional-mechanisms and iconic-memory mechanisms point of view, the after-coming singleton presented from the spatial location separated by slightly more than one degree of the visual angle from the target should aid selection from iconic memory and thus facilitate correct perception of target by acting as a valid after-cue. Why this expected effect did not take place, thereby contradicting hypothesis 5 , will be discussed later where we suggest possible mechanisms involved.

There are several problems with Experiment 1. First, the results on the adverse effect of the spatially close, but nonoverlapping after-cue are not very distinct and the highly significant effect of spatial separation can be attributed mainly to the condition of spatially overlapping targets and singletons (see Figure 2). It is therefore advisable to run an additional experiment where only spatially non-overlapping singletons are used in order to see whether the adverse effect of spatially close singletons as after-cues will stay. Second, when in many trials targets are masked by superimposed singletons as strong object masks, subjects may have learned that distinctly different singleton objects are detrimental for target perception (in these strong masking trials they rarely experience targets in their awareness) and because of this they become biased against trying to use singletons as attentional cues. They may have developed inherent "negative" attentional control settings "wary" of yellow singleton cues. At the same time this also may have increased the probability of target localization errors stemming from intrusion effects of distractors from non-target locations (e.g., see Chastain, 1990, about mislocalization as a 
common source of errors). For these reasons, Experiment 2 was run where only spatially non-overlapping singletons were used. We also increased the number of participants in order to test for the robustness of the effects.

\section{Experiment 2}

The aims of Experiment 2 were to replicate the results of Experiment 1 using only spatially separated singletons and targets in the conditions where subjects do not expect overlapping objects masking and with a larger number of subjects. Otherwise, the design, variables and hypotheses remain the same, except that we could not test the hypothesis about strong masking with spatially overlapping singletons and targets.

\section{Method}

\section{Participants}

Ten subjects participated (mean age 25, 4 females, 6 males). They were paid volunteers (180 Estonian Kroon per experiment per subject) who had normal or corrected-to-normal vision.

\section{Stimuli and Apparatus}

All stimuli and the apparatus were the same as in Experiment 1 , except that less singleton locations were used and singletons were never presented from the spatial locations superimposed with targets' and filler/distractors' locations. Both target and distracting items were selected from the same set of 8 stimuli as in Experiment 1; thus a varied mapping format was used again.

\section{Procedure}

The procedure was basically the same as in Experiment 1.
Feature-singletons appeared at randomly chosen locations relative to the target on an imaginary ring. The spatial distance between the singleton and target varyied between 1.55 deg, $4.42 \mathrm{deg}, 6.65 \mathrm{deg}$, and $7.84 \mathrm{deg}$ of visual angle. Each subject ran 1000 trials, with 10,000 trials across ten participants.

\section{Results and Discussion}

ANOVA was used to analyze of the effects of the factors of 1) SOA between target and masking singleton (11 levels: -120 , $-96,-72,-48,-24,0,24,48,72,96,120), 2)$ spatial distance between masking singleton and target (4 levels: 1.55 deg, 4.42 deg, $6.65 \mathrm{deg}$, and $7.84 \mathrm{deg}$ ), 3) target identity (8 levels). A repeated measures ANOVA shows that SOA had a moderately significant effect on correct target localization $(F(10,90)=2.11$, $p<.031$ ). As can be seen from Figure 3, this effect basically depends on the detrimental effect of the singleton that was presented more than $50 \mathrm{~ms}$ later than the target and only if presented from the closest spatial position from target. Consistent with this, the interaction between SOA and spatial distance was highly significant $(F(30,270)=2.26 ; p<.0003)$. Spatial distance between the singleton and target also had a highly significant effect that was also based on the conditions of closest spatial separation between target and singleton with positive SOA values $(F(3,27)=15.10, p<.00001)$ (see Figure $3)$. These results basically repeat the results of Experiment 1 in the conditions where singleton and Gabor stimuli do not spatially overlap and, particularly, substantiate that the only effect of the singleton emerges when it appears as an after-cue presented from the close spatial position to target. However, instead of the supposedly facilitative effect of the singleton

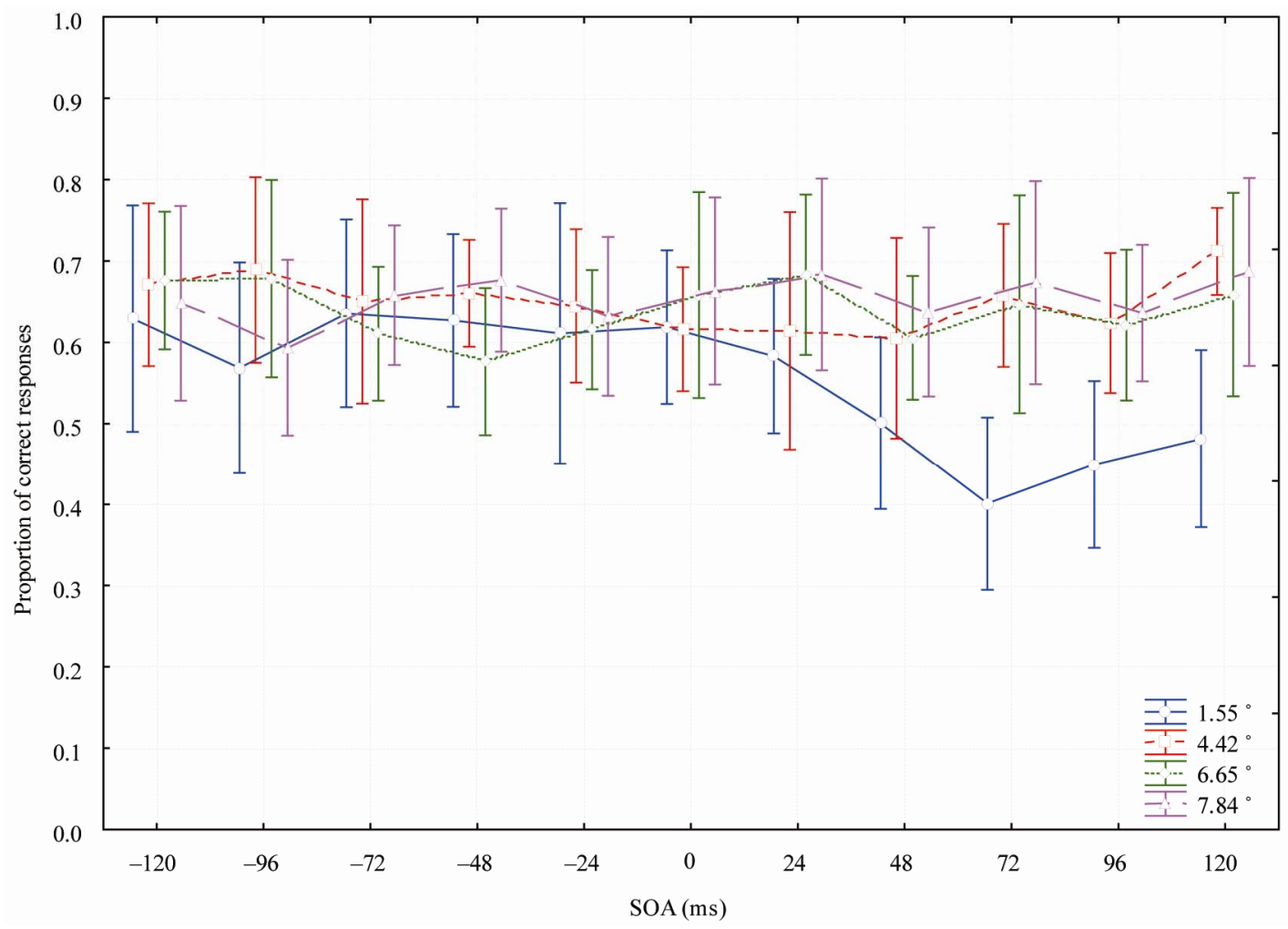

Figure 3.

Proportion of correct target localization responses as a function of SOA between uninformative singleton and target presentation and spatial distance between singleton and target. Only spatially close singletons presented 60 - $120 \mathrm{~ms}$ after target had a distractive effect. Vertical bars denote .95 confidence. (Data from Experiment 2.) 
expected to act as a selective attentional after-cue (to foster target selection from the iconic representation) there is an opposite effect - backward masking. There is also no facilitative precueing, no costly invalid precueing, no detrimental forward masking. (We would like also to note that the SOA values we used corresponded well to the optimal attentional pre-cueing SOAs in the range of about -50 to $-150 \mathrm{~ms}$ as found in earlier research-e.g., Cheal and Lyon, 1991.)

Introspective observations of subjects gathered after the experiment showed that in the trials where a nearby aftercoming singleton was effective in reducing target localization performance, often no explicit experience of the target was possible. Subjectively it appeared that the singleton substituted the nearby stimuli in awareness. Either mutual masking between objects (Bachmann, \& Allik, 1976; Hommuk \& Bachmann, 2009; Michaels, \& Turvey, 1979) or a new type of object substitution masking (Di Lollo et al., 2000; Enns, 2004) took place. The additional data about the percentages of errors of localization showed that subjects erred with roughly equal frequency whether their responded location was far from the actual target position or close to that position (percentages between 13.5 and 16.5 , ns). This means that it is unlikely that errors in target localization originate primarily from trials where target is explicitly perceived, but slightly mislocalized.

To sum up in interim: 1) a featurally and temporally uninformative singleton can impair target perception as measured by correct target localization, but only in limited experimental conditions, 2) the singleton effect appears even though the same singleton does not have any facilitatory or interfering (costly) effects of involuntary attentional capture on target localization (when presented as a pre-cue) and thus the masking may not originate directly from attentional mis-cueing, but appears as a complication in the process of building up explicit representation of the target, 3) the effect appears only when the singleton is presented more than $50 \mathrm{~ms}$ after target and from the nearest spatial position. No other significant main effects or interactions were found in this experiment. This pattern seems to be well accounted for by some unusual form of backward masking. However, it cannot be pattern masking because target and singleton do not overlap (and pattern masking is typically strongest with shortest target-to-mask SOAs-Bachmann, 1994). It is not also standard metacontrast masking because the spatial distance between target and singleton is too large (1.55 deg) and most of the target space is not surrounded by singleton elements. It is also difficult to accept the effect as object substitution masking (OSM) in its commonly accepted interpretation because the masks in the OSM paradigm have been metacontrast-like weak masks surrounding the target, with their effect depending on attention involvement. In our results, the backward object-masking effect was combined with insensitivity to attentional manipulations. Therefore, an option for interpretation would be either 1) to extend OSM effects to the ones where spatially neighbouring stimuli from as far as 1.55 degrees could intrude explicit attentive perception instead of the target or 2) to abandon the attentional explanation (that includes OSM as a theory critically dependent on whether selective attention can be deployed) and use some mechanism capable of depriving the target information from reaching conscious-level representation without directly invoking attention mechanisms. We come to theoretical discussion later on.

In experiments 1 and 2 the most compelling result besides strong forward and backward object masking was an effect of spatially remote lateral backward masking. But because we always used many distractor/filler objects we cannot be sure whether this effect originates primarily from close-range lateral interaction between target and the subsequent nearby singleton without any processing capacity or spatial-location uncertainty related effects or whether this new form of object masking critically depends on these higher level factors.

\section{Experiment 3}

In order to test directly whether a lateral-inhibitory masking of target identity features could be the main reason for the effects in Experiments 1 and 2, a simple identification experiment (Experiment 3) without distractors was run. We explored the effect of an uninformative feature-singleton (that was presented from different spatial locations never used for presentation of targets) on correct target identification as a function of spatial distance between singleton and target and on temporal separations between singleton and target. If local lateral inhibitory interaction between singleton and target is the principal or main cause of the effects found in the previous experiments, the close spatial distance between a singleton and a target should lead to a strong masking in this experiment as well. The basic design remained the same, but as the dependent measure rate of correct identification of targets was used. We test the hypothesis that when distractor objects are absent and attention must not be divided between alternative objects, the spatially close singleton will interfere with target processing, indicating a close-range lateral inhibition as the likely cause of the effects of Experiments 1 and 2.

\section{Method}

\section{Subjects}

Altogether 4 participants (mean age 35, two females, two males) participated. They had normal or corrected-to-normal vision.

\section{Stimuli and Apparatus}

Each stimulus used as a target was drawn from a set of 8 stimuli alternatives identical to those used in the previous experiments. In each trial one of the stimuli was presented for identification, located in one of the 8 locations forming an imaginary circle around the fixation dot (with a small radial jitter). Thus a target-stimulus appeared 3.8 - 4.3 degrees from fixation. The uninformative feature singleton for exerting lateral effects and for competing for bottom-up spatial attention with the target was set as a yellow ring singleton identical to the one used in the previous experiments. All of the equipment used was identical to that of the previous experiments.

\section{Procedure}

Each trial started with a presentation of the fixation cross $(.2$ deg). Participants initiated presentation of the stimuli by pressing the Enter key on the keyboard. After $1200 \mathrm{~ms}$, one of the three temporal types of trials occurred: a singleton appearing first, followed by the target appearing randomly in one of the 8 possible positions, with SOA varying between $120 \mathrm{~ms}, 96 \mathrm{~ms}$, $72 \mathrm{~ms}, 48 \mathrm{~ms}$, and $24 \mathrm{~ms}$; a target presented first, appearing randomly in one of the 8 possible positions, followed by a singleton with SOA varying between $120 \mathrm{~ms}, 96 \mathrm{~ms}, 72 \mathrm{~ms}, 48$ $\mathrm{ms}$, and $24 \mathrm{~ms}$; a target appearing randomly in one of the 8 possible locations and a singleton that was presented simultaneously with target. The singleton appeared in randomly chosen locations with the spatial distance between singleton and target varying randomly between the two distances of 1.55 deg or 7.84 deg of visual angle. After stimuli presentation, participants 
indicated by a mouse-click which one of the 8 possible targets was presented at that trial, guessing if necessary. Then next trial followed. Each subject ran between 540 and 960 trials, within a preset time of 60 minutes.

\section{Results and Discussion}

Data from each subject was subjected to an ANOVA for the analysis of the effects of the factors of 1) SOA between target and singleton (11 levels: $-120,-96,-72,-48,-24,0,24,48,72$, $96,120), 2)$ spatial distance between singleton and target (2 levels: 1.55 deg, $7.84 \mathrm{deg})$. Repeated measures ANOVA shows no effect of SOA $(F(10,30)=1.42, p<.218)$, there was an effect of the distance between target and singleton $(F(1,3)=$ 25.1, $p<.015$ ), but no interaction between SOA and distance showing that (and as different from the previous results) longer SOAs did not selectively lead to a stronger singleton effect $(F(10,30)=.88, p<.562)$. (See Figure 4 for the graph depicting the principal results.)

The suggested hypothesis predicted a difference in the level of identification between the conditions of close and far singleton-to-target separation stemming from the idea of lowlevel sensory lateral interaction between singleton and target. However, and importantly so with longer positive SOA values, increased interfering effect from distracting singleton when far and adjacent target-to-singleton distance conditions were compared, was absent. Local lateral interactions between the singleton and target are ineffective for selectively stronger impairment of target identification at specific SOAs. The uninformative singleton used in our experiments and having within-stimulus visual features different from the features of the targets (different unique singleton colour and absence of grating-features in the singleton contrary to the gratings with varying spatial frequencies and orientations used in targets) had neither selectively increased facilitating effects nor distracting effects as a function of the spatial distance from target. The correct identification rate at about $60 \%$ excludes both ceiling and floor effects. We conclude that a singleton used in our study can be consideread as a "weak" lateral backward mask when the possibility of local lateral interactions potentially effective on target identification were examined and when we assume insensitivity of the local early-level masking to the extent of attention distribution. (Notice the smaller number of the stimuli competing for attention in Experiment 3 compared to first two experiments.) At the same time this masking singleton was capable of strong masking effects in target location search in Experiments 1 and 2.

\section{General Discussion}

This study shows that a singleton object that is a powerful spatially overlapping visual mask in impairing target search among distractors does not have power to capture involuntary attention. This salient object that does not have power to influence visual target search when used as a singleton cue to capture attention (both as a valid cue expected to cause improvement and as an invalid cue expected to cause processing costs) nevertheless acts as a strong forward and backward mask when spatially overlapping with target. Importantly, the only condition where an uninformative salient singleton has a clear effect on target perception besides typical pattern/object masking is

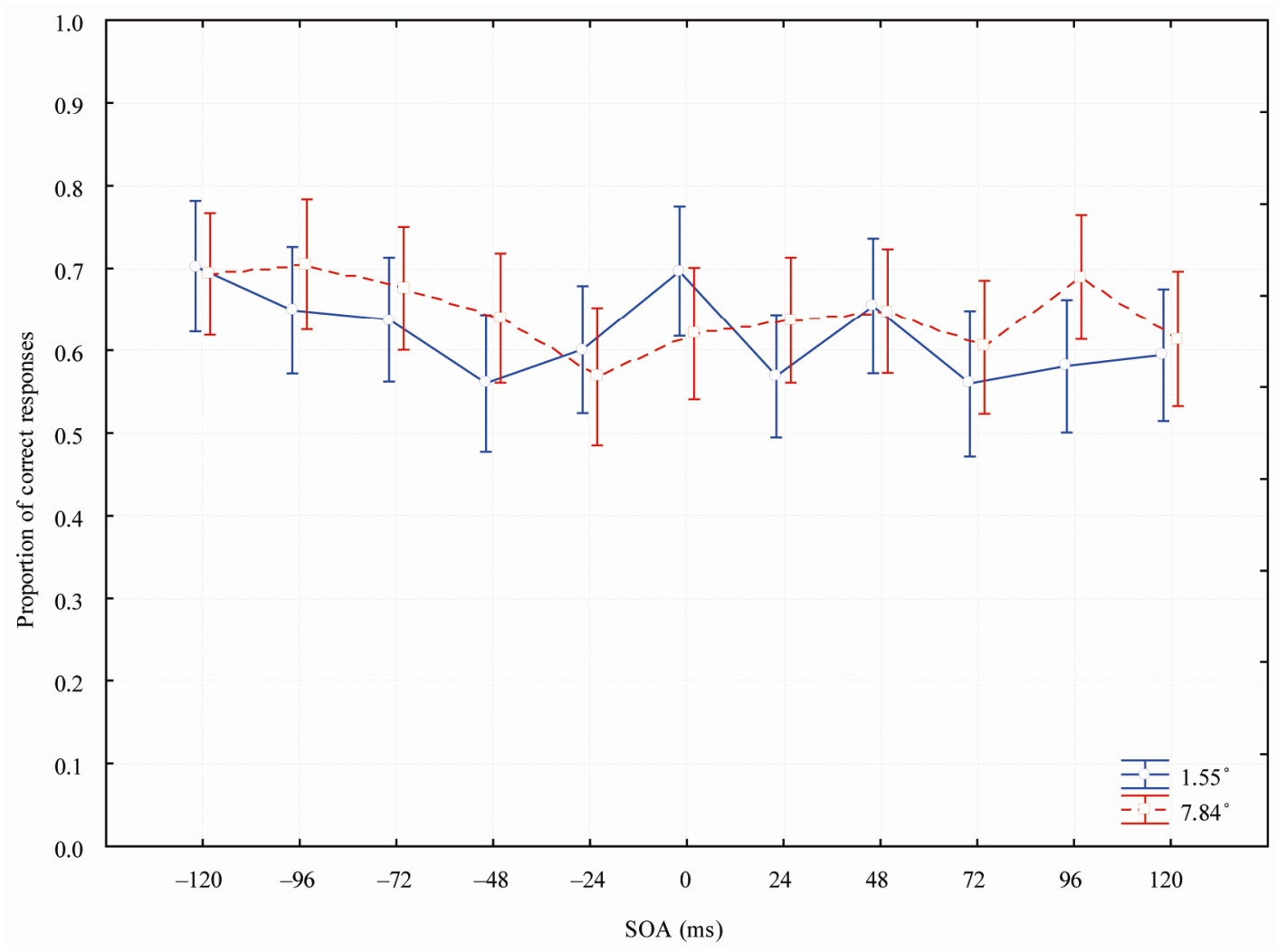

Figure 4.

Proportion of correct target identification responses as a function of SOA between uninformative singleton and target and spatial distance between singleton and target, averaged over 4 subjects. Correct target identification rate is same whether singleton was presented adjacent to target or from a far location with longer SOAs. (Data from Experiment 3.) 
when it is presented not far from the target and after it in time. Notably, this salient but irrelevant object can be easily ignored in a simple target identification task when no distractor elements are accompanying the target, but it has a strong adverse effect in the visual search task with the same target objects when their correct location has to be specified and filler/distractor items are presented simultaneouslyt with target. Therefore, the explanation for the spatially remote masking effect from the singletons that appear 50 - $100 \mathrm{~ms}$ after the target excludes early-level lateral inhibition.

For a spatially non-overlapping target and an irrelevant object there is no mutual interference with SOA set at zero or with negative SOAs. This means that an irrelevant singleton does not necessarily impair finding the target when presented just adjacent to it. Also, it does not interrupt search when presented after the target, but far from its spatial position. (Nor does it facilitate target processing when presented before it in time and near to it in space, as would be the case when it would act like an effective, valid, pre-cue.)

At first sight the results found here seem to suggest an interpretation quite similar to what is used for explaining the standard substitution masking (DiLollo, Enns, \& Rensink, 2000; Enns, 2004) and some other backward masking phenomena where masking strength is determined not only by the low-level visual-spatial and temporal relations between target and mask, but also by the dynamics of attention (e.g. Smith, 2000; Smith, \& Wolfgang, 2004). Similarly to standard substitution-masking which is studied with selective spatial attention controlled and manipulated, target loss from explicit perception as found in the present study may appear to presuppose an unfinished job of focusing spatial attention in obtaining the masking effects. However, as the singleton did not have any attentional cueing effects, its attention-capturing power can be doubted and therefore we may need to consider involvement of the mechanisms other than attention. A mechanism responsible for upgrading the pre-conscious target representation to explicit consciouslevel representation would be a good candidate. This is especially so because recent research has shown independence or even opposite effects of attention and consciousness-e.g., Koch and Tsuchiya, 2007; Bachmann and Murd, 2010. Many distractor elements set the stage for the singleton mask to have its effect and do this by depriving the target from the service by the consciousness mechanism, the target becoming masked by the singleton when it is close in space and follows the target.

The results show that stimulus identification mechanisms and spatial selection mechanisms that are needed to localize preattentively discriminated targets are at least partly autonomous. If a spatial selection operation that ultimately serves explicit perception would be dependent on feature-specific analysis, singletons should not get an advantage over targets because their features are clearly discriminable and different. It is possible to discuss the results from the point of view of possible beneficial selective spatial attentional effects mediated by spatially localized singletons and also from the perspective of possible interfering/inhibiting (costly) effects of the same singletons. Our results show that singletons that exert neither icreased local lateral sensory effects (either disturbing or facilitating ones) nor invalid pre-cueing costs on target identification do exert disturbing effects in the conditions where a preknown target has to be found among distractor items and correctly localized. This effect comes about only retroactively, i.e., when targets and distractors have been already presented. In this case a singleton that in principle can be ignored as a competing item because of its consistently different featural identity in the identification task cannot be ignored as a token that competes with the target for their location to be specified.

Uninformative singletons that are presented close to or overlapping with subsequent targets did not facilitate target processing in the search/localization task. (Neither did spatially remote singletons produce any invalid cueing costs.) This makes our experimental conditions compatible with the requirements needed to satisfy the principle that uninformative singletons are inefficient as attention-capturing stimuli (e.g., Becker, 2007; Gibson et al., 2008; Yantis \& Jonides, 1990; Jingling \& Yeh, 2007). This state of affairs does not mean that singletons can be absolutely ignored or made ineffective due to some suitable control setting. Our results stress that in order to claim absence of any singleton effects on target processing a systematic change in spatial and temporal values of intervals between targets and singletons has to be used. This is because we have found a highly selective singleton effect in space and time. When a post-target singleton appears close to a target that is pre-attentively processed but, in terms of its featuresexplicitly unidentified, the singleton disturbs target processing by substituting it or by making an obstacle for explicit locationbound identification of it in the conscious representation. Our present experiments do not specify whether this interference takes place at the parallel stage of processing or at some stage of serial processing when alternative items are analysed for a match with the target cues. However, it has to occur before the target has been explicitly localised by being experienced in consciousness.

In guided search models (Hoffman, 1979; Wolfe, Cave, \& Franzel, 1989) it is possible to envisage a scenario where the distracting singleton interferes at the unfinished parallel preattentive stage of processing by substituting the target (akin to singleton pop-out) or at the stage of subsequent focal selection of an item to be responded to where, again, the singleton can substitute for the target and indicate to an observer that this location of interest, "unfortunately", contains an irrelevant stimulus. (The priority of spatial location over other attributes in top-down controlled visual search is supported, e.g., by Grabbe and Pratt, 2004; Kim and Cave, 1999.)

The time course of the interference effect suggests that the target's appearance in explicit perception with its actual location being experienced cannot be generated very fast: the aftercoming singleton's interfering effect on target perception extended to more than $100 \mathrm{~ms}$. It is therefore not surprising that single-cell studies of stimulus pop-out also indicate that at least 70 - $230 \mathrm{~ms}$ post-target time appears to be necessary for a secondary, top-down effect of target enhancement (e.g., Smith, Kelly, \& Lee, 2007). Moreover, the recent work on localized attentional interference between neighboring visual-object representations also showed that spatial distance takes its effect mostly with longer target-to-mask/cue SOAs (Steelman-Allen, McCarley, \& Mounts, 2009). However, in the present study the objects that do not show any capability to invoke spatialattentional capture facilitating target perception or impairing target perception by effective misdirection of selective attention nevertheless influence explicit perception of targets in their correct location. Yet another attentional mechanism to be considered for explaining our results is related to centersurround profile of the focus of attention (e.g., Boehler, Tsotsos, Schoenfeld, Heinze, \& Hopf, 2009). In this case, however, there are two problems. First, the optimal delay for the topdown controlled and temporally delayed surround attenuation effects (i.e., formation of the inhibitory area around the singleton in our experiment) was shown to be more than $175 \mathrm{~ms}$, 
which is by far too slow an effect compared to our data. Second, this inhibitory surround effect has been related to featurebinding operations, but our singleton is uninformative and does not share varied-mapping features with targets. Third and most importantly, our adverse effect of singleton on target perception was found when the singleton followed the target in time and therefore target must have been inhibiting the later-coming singleton and not vice versa.

Recently, Munneke, Van der Stigchel and Theeuwes (2008) showed that an irrelevant distractor can be made less efficient by a top-down inhibitory mechanism that helps to better ignore the irrelevant onset-stimulus. But even then the object-substitution effects strongly interfered with the target's explicit identification, even though the relevant spatial location has been already successfully established. This means that in addition to identity processing, visual awareness also requires correct localization of the target stimulus within the map of stimuli locations. It is even likely that, in principle, there cannot be distinct visual awareness at all if an identified object has not been granted its stable spatial position within the microgenetically evolving subjective perceptual image (e.g., Bachmann, 1994).

Taken together, the present study demonstrates a new version of masking in visual search where the need for features-based guidance of attentional search among the varied-mapping alternatives makes the target vulnerable from an otherwise ineffective masker. The singleton can substitute the target also from a spatially shifted position, leaving the target often out of conscious experience.

\section{Acknowledgements}

Support provided by Estonian Ministry of Education and Research through Scientific Competency Council (targeted financing research theme SF0182717s06, "Mechanisms of Visual Attention”) is very much appreciated.

\section{References}

Ansorge, U., \& Horstmann, G. (2007). Preemptive control of attentional capture by colour: Evidence from trial-by-trial analyses and orderings of onsets of capture effects in reaction time distributions. Quarterly Journal of Experimental Psychology, 60, 952-975. doi:10.1080/17470210600822795

Bachmann, T. (1994). Psychophysiology of visual masking. The fine structure of conscious experience. Commack, New York: Nova Science Publishers.

Bachmann, T., \& Allik, J. (1976). Integration and interruption in the masking of form by form. Perception, 5, 79-97. doi:10.1068/p050079

Bachmann, T., \& Murd, C. (2010). Covert spatial attention in search for the location of a color-afterimage patch speeds up its decay from awareness: Introducing a method useful for the study of neural correlates of visual awareness. Vision Research, 50, 1048-1053. doi:10.1016/j.visres.2010.03.013

Bachmann, T., Mäger, K., Sarv, M., Kahusk, N., \& Turner, J. (1999). Time-course of spatial-attentional focusing in the case of high processing demand on the peripheral precue. European Journal of Cognitive Psychology, 11, 167-198. doi:10.1080/713752312

Becker, S. I. (2007). Irrelevant singletons in pop-out search: attentional capture or filtering costs? Journal of Experimental Psychology: Human Perception and Performance, 33, 764-787. doi:10.1037/0096-1523.33.4.764

Boehler, C. N., Tsotsos, J. K., Schoenfeld, M. A., Heinze, H.-J., \& Hopf, J.-M. (2009). The center-surround profile of the focus of attention arises from recurrent processing in visual cortex. Cerebral Cortex, 19, 982-991. doi:10.1093/cercor/bhn139
Breitmeyer, B. G. (2010). Blindspots. The many ways we cannot see. Oxford, New York: Oxford Universitgy Press.

Chastain, G. (1990). Representation of letters when mislocation errors occur. Journal of General Psychology, 117, 143-151. doi:10.1080/00221309.1990.9921131

Cheal, M. L., \& Lyon, D. R. (1991). Central and peripheral precuing of forced-choice discrimination. Quarterly Journal of Experimental Psychology, 43, 859-880. doi:10.1080/14640749108400960

Davoli, C. D., Suszko, J. W., \& Abrams, R. A. (2007). New objects can capture attention without a unique luminance transient. Psychonomic Bulletin and Review, 14, 338-343. doi:10.3758/BF03194074

Di Lollo, V., Enns, J. T., \& Rensink, R. A. (2000). Competition for consciousness among visual events: the psychophysics of re-entrant visual processes. Journal of Experimental Psychology: General, 129, 481-507. doi:10.1037/0096-3445.129.4.481

Enns, J. T. (2004). Object substitution and its relation to other forms of visual masking. Vision Research, 44, 1321-1331. doi:10.1016/j.visres.2003.10.024

Folk, C. L., Remington, R. W., \& Johnston, J. C. (1992). Involuntary covert orienting is contingent on attentional control settings. Journal of Experimental Psychology: Human Perception and Performance, 18, 1030-1044. doi:10.1037/0096-1523.18.4.1030

Forster, S., \& Lavie, N. (2008). Attentional capture by entirely irrelevant distractors. Visual Cognition, 16, 200-214. doi:10.1080/13506280701465049

Gibson, B. S., Folk, C., Theeuwes, J., \& Kingstone, A. (Eds.) (2008). Attentional capture. Hove: Psychology Press.

Grabbe, Y., \& Pratt, J. (2004). Competing top-down processes in visual selection: Evidence that selection by location is stronger than selection by color. Journal of General Psychology, 131, 137-149. doi:10.3200/GENP.131.2.137-150

Hoffman, J. E. (1979). A two-stage model of visual search. Perception \& Psychophysics, 25, 319-327. doi:10.3758/BF03198811

Hommuk, K., \& Bachmann, T. (2009). Temporal limitations in the effective binding of attended target attributes in the mutual masking of visual objects. Journal of Experimental Psychology: Human Perception and Performance, 35, 648-660. doi:10.1037/a0013481

Jingling, L., \& Yeh, S.-L. (2007). New objects do not capture attention without a top-down setting: evidence from an inattentional blindness task. Visual Cognition, 15, 661-684 doi:10.1080/13506280600926695

Kahneman, D., \& Treisman, A. (1984). Changing views of attention and automaticity. In R. Parasuraman and D. R. Davis (Eds.), Varieties of attention (pp. 29-61). Orlando: Academic Press.

Kawahara, J., \& Miyatani, M. (2001). The effect of informative and uninformative cueing of attention on feature integration. Journal of General Psychology, 128, 51-75. doi:10.1080/00221300109598898

Kim, M. S., \& Cave, K. R. (1999). Grouping effects on spatial attention in visual search. Journal of General Psychology, 126, 326-352. doi:10.1080/00221309909595370

Koch, C., \& Tsuchiya, N. (2007). Attention and consciousness: two distinct brain processes. Trends Cognitive Sciences, 11, 16-22. doi:10.1016/j.tics.2006.10.012

Lichtenstein-Vidne, L., Henik, A., \& Safadi, Z. (2007). Task-relevance modulates the effects of peripheral distractors. Quarterly Journal of Experimental Psychology, 60, 1216-1226. doi:10.1080/17470210600917892

Michaels, C. F., \& Turvey, M. T. (1979). Central sources of visual masking: Indexing structures supporting seeing at a single, brief glance. Psychological Research, 41, 1-61. doi:10.1007/BF00309423

Mulckhuyse, M., \& Theeuwes, J. (2010). Unconscious attentional orienting to exogenous cues: A review of the literature. Acta Psychologica, 134, 299-309. doi:10.1016/j.actpsy.2010.03.002

Munneke, J., Van der Stigchel, \& Theeuwes, J. (2008). Cueing the location of a distractor: An inhibitory mechanism of spatial attention? Acta Psychologica, 129, 101-107. doi:10.1016/j.actpsy.2008.05.004

Müller, H. J., \& Krummenacher, J. (Eds.) (2006). Visual search and attention. Hove: Psychology Press.

Neo, G., \& Chua, F. K. (2006). Capturing focused attention. Perception \& Psychophysics, 68, 1286-1296. doi:10.3758/BF03193728

Smith, M. A., Kelly, R. C., \& Lee, T. S. (2007). Dynamics of response to perceptual pop-out stimuli in macaque V1. Journal of Neurophysiology, 98, 3436-3449. doi:10.1152/jn.00441.2007 
Smith, P. L. (2000). Attention and luminance detection: Effects of cues, masks, and pedestals. Journal of Experimental Psychology: Human Perception and Performance, 26, 1401-1420.

doi:10.1037/0096-1523.26.4.1401

Smith, P. L., \& Wolfgang, B. J. (2004). The attentional dynamics of masked detection. Journal of Experimental Psychology: Human Perception and Performance, 30, 119-136.

doi:10.1037/0096-1523.30.1.119

Steelman-Allen, K., McCarley, J. S., \& Mounts, J. R. W. (2009). Localized attentional interference reflects competition for reentrant processing. Psychonomic Bulletin \& Review, 16, 110-115. doi:10.3758/PBR.16.1.110

Turatto, M., \& Galfano, G. (2000). Color, form and luminance capture attention in visual search. Vision Research, 40, 1639-1643. doi:10.1016/S0042-6989(00)00061-4

Van den Bussche, E., Hughes, G., Van Humbeeck, N., \& Reynvoet, B. (2010). The relation between consciousness and attention: An em- pirical study using the priming paradigm. Consciousness and Cognition, 19, 86-97. doi:10.1016/j.concog.2009.12.019

Vierck, E., \& Miller, J. (2008). Precuing benefits for color and location in a visual search task. Perception \& Psychophysics, 70, 365-373. doi:10.3758/PP.70.2.365

Wolfe, J. M., Cave, K. R., \& Franzel, S. L. (1989). Guided search: An alternative to the feature integration model for visual search. Journal of Experimental Psychology: Human Perception and Performance, 15, 419-433. doi:10.1037/0096-1523.15.3.419

Yantis, S., \& Jonides, J. (1990). Abrupt visual onsets and selective attention: voluntary versus automatic allocation. Journal of Experimental Psychology: Human Perception \& Performance, 16, 121-134. doi:10.1037/0096-1523.16.1.121

Yeh, S.-L., \& Liao, H.-I. (2010). On the generality of the displaywide contingent orienting hypothesis: Can a visual onset capture attention without top-down control settings for displaywide onset? Acta Psychologica, 135, 159-167. doi:10.1016/j.actpsy.2010.05.013 